\title{
Influence du substrat sur la répartition des macroinvertébrés benthiques dans un système lotique : cas des rivières Gombe, Kinkusa et Mangengenge
}

\author{
Jean-Claude KAMB TSHIJIK ${ }^{1 *}$, Séraphin NDEY IFUTA ${ }^{2}$, \\ Alexandre NTUMBULA MBAYA ${ }^{1}$ et Victor KIAMFU PWEMA ${ }^{3}$ \\ ${ }^{1}$ Université Pédagogique Nationale (UPN), Faculté des Sciences, Département de Biologie, B.P. 8815 \\ Kinshasa / Ngaliema, R.D Congo. \\ ${ }^{2}$ Institut Supérieur Pédagogique de la Gombe (ISP/Gombe), R.D Congo. \\ ${ }^{3}$ Laboratoire Limnologie, Hydrobiologie et Aquaculture (LLHA), Université de Kinshasa, Faculté des \\ Sciences, Département de Biologie, B.P. 190 Kinshasa XI, R.D Congo. \\ *Auteur correspondant, E-mail : jckamb881962@gmail.com
}

\section{RESUME}

La répartition géographique des espèces dans un écosystème est liée à leurs habitats. L'influence du substrat sur la distribution des macroinvertébrés benthiques de trois hydrosystèmes de la ville de Kinshasa (Gombe, Kinkusa et Mangengenge) était étudiée de mai en début septembre 2014. L'échantillonnage des macroinvertébrés était effectué grâce au filet troubleau dans chaque type de substrat. Douze types de substrats étaient regroupés en deux grandes catégories : la vase et le sable. Le gravier ayant été présent en faible proportion. Cinq mille huit cent quatre-vingt et un macroinvertébrés appartenant à trente deux familles ont été récoltés : seize familles ont été récoltés respectivement dans la Gombe et dans la Kinkusa; et vingt quatre dans la Mangengenge. Seize familles étaient localisées dans le sable et le gravier, quinze dans la vase et cinq étaient concomitamment présentes dans le sable et la vase. La présence des Trichoptères dans la Mangengenge associée aux valeurs indiciaires de Shannon et Weaver $(3,6)$ et à la note de l'Indice Biologique Général Normalisé (IBGN) (14/20), montre que l'eau de cet hydrosystème est de bonne qualité par rapport à celles des rivières Gombe et Kinkusa qui sont polluées et similaires à $73 \%$.

(C) 2015 International Formulae Group. All rights reserved.

Mots clés : Kinshasa, rivières, substrat, influence, macroinvertébrés benthiques.

\section{INTRODUCTION}

La ville province de Kinshasa, construite sur la rive gauche du fleuve Congo, dispose d'un important réseau hydrographique constitué essentiellement des rivières d'importances, variables (Lelo, 2008). Malgré cet important réseau hydrographique, ces cours d'eaux comme ceux qui traversent les grandes villes africaines sont utilisés pour l'arrosage des cultures maraîchères, la baignade, la lessive et servent également à l'élimination des effluents domestiques et industriels (Alhou, 2007). Ces multiples usages de l'eau altèrent sa qualité et perturbent l'équilibre de la biocénose locale ainsi que le fonctionnement général de cet écosystème (Brusle et Quignard, 2004).

La surveillance biologique est un élément essentiel pouvant servir à évaluer la qualité d'un cours d'eau (Piscart et al., 2005). Dans ce système, les macroinvertébrés benthiques sont considérés comme de bons indicateurs de la santé des écosystèmes aquatiques à cause de leur sédentarité, leur cycle de vie varié, leur grande diversité et leur tolérance variable à 
la pollution et à la dégradation de l'habitat (Moisan et Pelletier, 2008 ; Diomande et al., 2009; Foto et al., 2011 ; MDDEFP, 2013).

En République Démocratique du Congo en général et à Kinshasa en particulier, les peuplements des macroinvertébrés benthiques restent mal connus malgré quelques études menées par Mbadu (2002), Kabamba (2003), Puta (2005), Tubi (2005), Mangaya (2010), Kuyitila (2011) et Kamb (2013) respectivement dans les rivières Lukunga, Maluku, Kwambila, Bumbu, Tshwenge, N'sele et Mango. Toutefois, la relation intime entre les macroinvertébrés benthiques et les sédiments qui constituent les différents substrats de ces différents cours d'eaux, y est peu élucidée.

Le présent travail est donc une contribution à la connaissance de la structure des peuplements des macroinvertébrés benthiques en relation avec les substrats de fond de quelques hydrosystèmes de la ville de Kinshasa.

\section{MATERIEL ET METHODES \\ Milieu d'étude}

Trois rivières de la ville province de Kinshasa ont été étudiées. Il s'agit de : Gombe, Kinkusa et Mangengenge. Les coordonnées géographiques de ces trois cours d'eaux sont présentées dans le Tableau 1.

La rivière Gombe traverse le centre ville de Kinshasa d'Est à l'Ouest et charrie une quantité importante de déchets domestiques et industriels sur une distance de $9 \mathrm{~km}$.

La rivière Kinkusa prend sa source dans le versant de L'Université Pédagogique Nationale du Congo et parcourt une zone en plein lotissement présentant encore de galeries forestières. Elle coule du Sud-Est vers le Nord-Ouest sur une distance de $8 \mathrm{~km}$ et transporte les déchets domestiques avant de se jeter dans le fleuve Congo.

La rivière Mangengenge, située en périphérie de la ville de Kinshasa, dans une zone non perturbée par les activités anthropiques, prend sa source dans les hautes montagnes qui portent son nom et coule d'Est vers l'Ouest sur une longueur de $5 \mathrm{~km}$ et, est un affluent du le fleuve Congo (Figure 1).

Selon Bultot (1954), Kinshasa baigne dans un climat tropical chaud et humide de type Aw4 suivant la classification de Köppen.
Deux grandes saisons caractérisent le climat de cette région: la saison sèche qui s'étale sur trois mois (juin, juillet et août) et celle pluvieuse qui prend neuf mois (septembre à mai). Une courte saison sèche s'intercale entre janvier et février. Toutefois, compte tenu des mutations climatiques que connaît la région de Kinshasa, ce système de classification nécessite une mise à jour (Kamb, 2013).

\section{Caractéristiques des particules sédimentaires}

Le prélèvement d'échantillons de substrat était effectué dans chaque station à une profondeur de $10 \mathrm{~cm}$ à l'aide d'une tarière. L'analyse de la taille des particules colloïdales a été effectuée au laboratoire où les échantillons ont subi un tamisage afin de séparer la vase du sable. Cette méthode nous a conduit à définir les sédiments non biogènes de façon qualitative.

\section{Echantillonnage des macroinvertébrés benthiques \\ Cinq milles huit cent quatre vingt et} un (5.881) macroinvertébrés benthiques ont été échantillonnés pendant la saison sèche (mai, juin, juillet août et septembre) en 30 campagnes, selon une périodicité bimensuelle par rivière dans 14 stations différentes. Trois milles sept cent trente et un (3.731) macroinvertébrés ont été récoltés dans la rivière Gombe, mille sept cent quatre-vingt-treize (1793) dans la rivière Kinkusa et trois cent cinquante sept (357) dans la rivière Mangengenge. L'échantillonnage a été fait à l'aide d'un troubleau de $30 \mathrm{~cm}$ X $30 \mathrm{~cm}$. Lors de l'échantillonnage, l'ouverture $\mathrm{du}$ filet est placée à contre-courant et le filet est traîné légèrement sur le fond le long d'un transect de $100 \mathrm{~m}$ de long en passant par le maximum d'habitats possibles. Le filet est ensuite relevé et le contenu bien concentré est reversé dans des bocaux contenant du formol $10 \%$. Le tri est effectué plus tard au laboratoire.

L'identification des spécimens a été effectuée à l'aide des clés de détermination proposées par Durand et Lévêque, (1981), Moisan (2010) et Tachet et al. (2010), sous une stéréo-loupe binoculaire et, 
occasionnellement nous avons eu recours au microscope pour certains détails précis.

Afin de réduire le risque d'erreur, la position systématique a été faite jusqu'au niveau de la famille. Ceci étant utile lorsqu'on veut obtenir un portrait général d'un écosystème aquatique (Touzin, 2008). Le niveau d'identification requis, qui est majoritairement la famille ou le genre, offre donc la possibilité d'utiliser les macroinvertébrés benthiques de façon efficace, fiable et peu coûteuse afin de déterminer le degré de dégradation des rivières (Touzin, 2008).

\section{Traitement des données biologiques}

La structure des peuplements des macroinvertébrés benthiques a été étudiée à l'aide de la richesse taxonomique et de l'abondance relative. Pour évaluer l'impact d'une éventuelle modification du milieu sur les macroinvertébrés benthiques, plusieurs indices ont également été calculés. Il s'agit de: - l'indice de diversité de Shannon et Weaver : Cet indice est calculé à partir des listes faunistiques obtenues selon la formule suivante (Evrard, 1996) :

$H^{\prime}=-\sum_{i=1}^{S} p_{i} \log _{2} p_{i}$

Où, $\mathrm{H}^{\prime}$ : indice de biodiversité de Shannon $i$ : une espèce du milieu d'étude

$p_{i}$ : Proportion d'une espèce $i$ par rapport au nombre total d'espèces $(S)$ dans le milieu d'étude (ou richesse spécifique du milieu), qui se calcule de la façon suivante :

$$
p(i)=n_{i} / N
$$

Où $n_{i}$ est le nombre d'individus pour l'espèce $i$ et $\mathrm{N}$ est l'effectif total (les individus de toutes les espèces).

Dans la nature, la valeur de H' se situe entre 0,5 (très faible diversité) et 4,5 (dans le cas d'échantillons de grande taille de communautés complexes). Du point de vue de la pollution, cet indice permet de distinguer trois classes de qualité des eaux (Evrard, 1996) : a. $2 \leq H^{\prime}$ pour une eau propre ; b. $1<$ $H^{\prime}<2$ pour une eau modérément polluée ; b. $H^{\prime} \leq 1$ pour une eau polluée.

- l'indice d'équitabilité de Piélou : Il mesure l'équilibre du peuplement. C'est le rapport de
$\mathrm{H}^{\prime}$ sur $\mathrm{H}^{\prime}{ }_{\max }$. Cet indice varie de 0 à 1 . Il est maximal quand les taxons du peuplement ont des abondances identiques. Il tend vers 0 quand la quasi-totalité des effectifs est concentrée sur un seul taxon.

- l'indice de similarité de Sørensen. Il permet la comparaison faunistique des rivières (Sørensen, 1948). Il est obtenu selon la relation suivante :

$$
C s=\frac{2 c}{a+b} * 100
$$

Où : Cs : coefficient de similitude de Sørensen ; a : nombre de taxon dans le milieu A

b : nombre de taxon dans le milieu B; c : nombre de taxon commun à $\mathrm{A}$ et $\mathrm{B}$.

- l'indice Hilsenhoff (FBI): Il permet d'apprécier le degré de pollution d'un cours d'eau ; et est calculé à partir de la relation suivante (Hilsenhoff, 1988):

$$
F B I=\frac{\sum x i * t i}{n}
$$

Où : x $\mathrm{i}$ : nombre d'individus du $\mathrm{i}^{\text {ème }}$ taxon ; ti :tolérance du ième taxon; $\mathrm{n}$ : nombre d'individus composant l'échantillon et FBI : indice de Hilsenhoff. L'échelle d'interprétation des résultats est reprise au Tableau 2.

- l'indice Biologique Global Normalisé (IBGN) (Rodier et al., 2009). Il est noté sur 20 et a été déterminé conformément aux prescriptions reprises dans la norme NF T 90-350 de mars 2004 d'après AFNOR (2004).

\section{Analyses statistiques des données}

L'analyse factorielle des correspondances (AFC) est utilisée pour déterminer les différentes communautés de macroinvertétébrés le long des rivières étudiées après une transformation logarithmique de type LOG $(\mathrm{X}+1)$. L'analyse de classification hiérarchique ou analyse de regroupement (cluster analysis) est également utilisé pour regrouper les rivières selon la richesse taxonomique (Legendre et Legendre, 1998). Le logiciel Past (Paleontological statistics, Version 2.16) a été utilisé à cet effet (Hammer et al., 2001). 


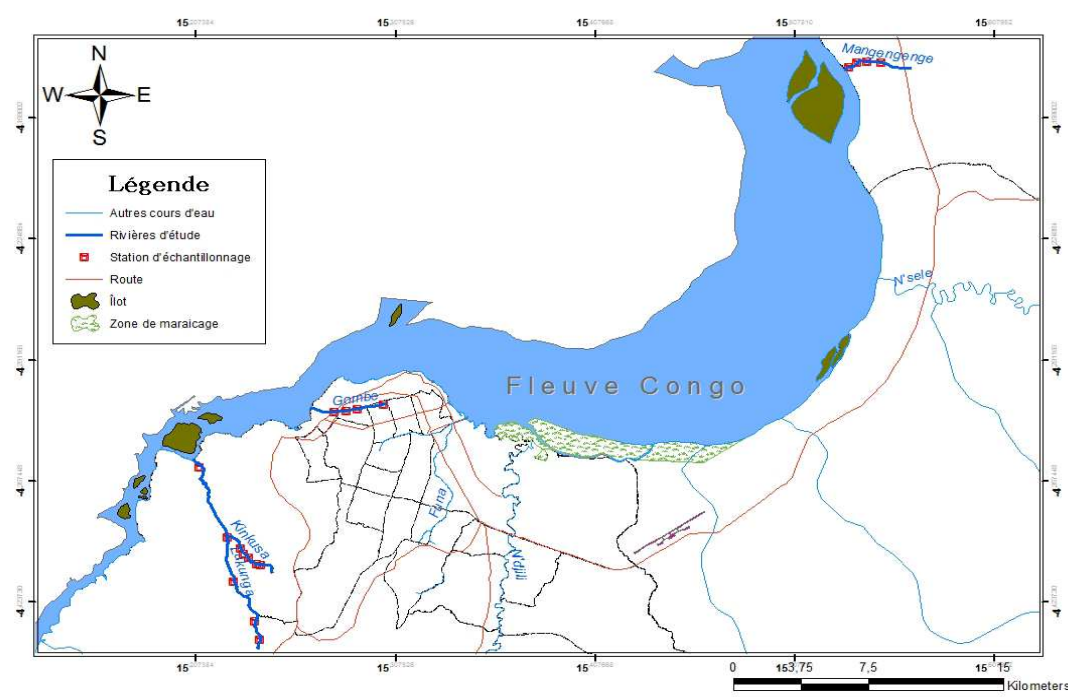

Figure 1 : Localisation des rivières et stations étudiées dans la ville de Kinshasa.

\section{RESULTATS}

Evaluation des habitats benthiques des Macroinvertébrés

Les habitats qui composent les lits des trois rivières à chaque station, ont été quantifiés et classés en 12 grandes catégories (Tableau 3). Ce sont des combinaisons de vase, de sable, de végétation émergente, de galet et du gravier.

L'analyse factorielle des correspondances (AFC) réalisée sur les données des macroinvertébrés récoltés dans chaque type de substrat montre que ces stations sont subdivisées en trois groupes (Figure 2). Cette analyse explique $74,51 \%$ de variabilité observée dont $45,36 \%$ par l'axe 1 et $29,15 \%$ par l'axe 2 . Les valeurs propres étant de 14,51 et 9,33 respectivement pour l'axe 1 et 2 . Le substrat de type L (composé $\mathrm{du}$ gravier, de sable et de matière organique) et le substrat de type $\mathrm{C}$ (composé de vase, de matière organique et de végétation émergente) se démarquent des autres substrats.

Les macroinvertébrés benthiques ont été échantillonnés dans six types de substrats dans la rivière Gombe. Ces substrats sont des combinaisons des sédiments et de la végétation émergente. La vase associée à la matière organique et à la végétation émergente a constitué l'essentiel des substrats dans toutes les stations étudiées dans ce cours d'eau. Dans la rivière Kinkusa, on note la prépondérance du substrat constitué du sable associé à la matière organique et à la végétation émergente. La rivière Mangengenge est caractérisée par une présence du gravier associé au sable et à la végétation émergente, et par une faible présence de la vase associée à la matière organique.

\section{Diversité taxonomique}

Cinq mille huit cent quatre-vingt et un (5.881) macroinvertébrés appartenant à 7 ordres et 32 familles ont été récoltés. Il s'agit des Hémiptères, des Odonates, des Coléoptères, des Diptères, des Clitellates, des Trichoptères et des Gastéropodes (Tableau 4).

Les insectes ont été les plus abondants (75\%), suivis des Clitellates (19\%) et des Gastéropodes (6\%) (Figure 3).

Ces organismes se répartissent de la manière suivante par rivière ;

- Gombe : Hémiptères (0,1\%),

Coléoptères $(1,3 \%)$, Clitellates $(25,5 \%)$, Diptères $(59,1 \%)$, Trichoptères $(1,6 \%)$ et Gastéropodes $(12,4 \%)$;

- Kinkusa: Hémiptères (8,4\%), Coléoptères $(1,2 \%)$, Clitellates $(64,8 \%)$, Diptères $(0,9 \%)$, Gastéropodes $(0,9 \%)$, Odonates $(23,4 \%)$, et Trichoptères $(0,4 \%)$;
- Mangengenge :
Hémiptères

$(28,1 \%)$, Coléoptères $(10,9 \%)$, Clitellates 
$(2,5 \%)$, Diptères $(0,8 \%)$, Odonates $(43,1 \%)$ et Trichoptères $(14,6 \%)$.

Les Odonates et les Trichoptères qui sont généralement cités parmi les groupes sensibles à la pollution (Muli et Mavuti, 2001) totalisent 10 familles, soit $57,7 \%$ de la richesse taxonomique de la rivière Mangengenge, 4 familles, soit 23,3\% de la richesse taxonomique de la rivière Kinkusa et 1 famille, soit $1,6 \%$ de la richesse taxonomique de la rivière Gombe. Les Chironomidae et les Mollusques considérés comme polluorésistants totalisent 3 familles dans la rivière Gombe, 2 dans la rivière Kinkusa et aucune dans la rivière Mangengenge. Il faut également signaler la présence des Syrphidae dans les rivières Gombe et Kinkusa qui est une famille de Diptère caractéristique des milieux fortement pollués.

L'analyse en composantes principales représentée par la Figure 4 a permis d'aboutir à une distribution des taxons en forme de croissant dans le plan formé par les axes 1 et 2. Ces derniers expliquent $53,2 \%$ de la variabilité observée dont $34,6 \%$ par l'axe 1 et $18,6 \%$ par l'axe 2. L'inertie totale est de 1,03. Ces macroinvertébrés sont répartis en trois groupes : Le premier groupe est composé des Lumbricidae, Tubificidae, Huridinidae, Lumbricidae et coenagronidae. Le deuxième groupe est composé des Libellulidae, Spercheidae et le troisième de Hydropshichidae, Glossossomatidae, Gomphidae et aphelocheilidae.

Six familles de macroinvertébrés benthiques sont communes aux trois rivières. Il s'agit de Nepidae, Culicideae, Lumbricidae, Huridinidae, Lumbriculidae et Hydrophilidae. Les familles des Elmidae et des Planariidae n'ont été observées que dans la rivière Gombe. Treize familles sont localisées dans la rivière Mangengenge. Il s'agit de : Potamantidae, Glossosomatidae, Philopotamidae, Leptoceridae, Ecnomidae, Naidideae, Dystsicidae, Limnebiidae, Gerridae, Aphelocheriidae, Notonectidae, Gomphidae, Ceratopogonidae.

Onze (11) familles des macroinvertébrés benthiques ont été récoltées uniquement dans des substrats contenant du sable. Il s'agit de : Aphelocheilidae, Gerridae, Notonectidae, Gomphidae, Dysticidae, Ceratopogonidae, Ecnomodae, Leptoceridae,
Glossossomatidae, Potamantidae et Philopotamidae (Tableau 5).

Cinq familles sont localisées dans les milieux contenant de la vase. Il s'agit de : Elmidae, Chironomidae, Planariidae, Limnebiidae et Limneidae.

Quinze familles sont communes aux deux grands groupes de substrats. Il s'agit de : Nepidae, Naucoridae, Coenagrionidae, Libellulidae, Hygrobiidae, Spercheidae, Hydrphilidae, Lumbricidae, Huridinidae, Lumbriculidae, Tubificidae, Culicidae, Syphidae, Hydropshychidae et Physidae.

Le Dendrogramme de similarité des rivières établi en fonction de la richesse taxonomique des macroinvertébrés benthiques de chacune d'elles, indique que ces cours d'eaux peuvent être réparties en deux groupes distincts $(\mathrm{r}=0,72)$. Les rivières Gombe et Kinkusa sont proches mais cellesci sont éloignées de la rivière Mangengenge (Figure 5).

Le Tableau 6 présente l'abondance des taxons dans les types de substrats étudiés.

\section{Évaluation indiciaire}

La valeur moyenne de l'indice de diversité de Shannon et Weaver est de $(1,4 \pm 0,12)$ dans la rivière Gombe, $(1,8 \pm 0,3)$ dans la rivière Kinkusa et $(3,3 \pm 0,36)$ dans la rivière Mangengenge.

La note attribuée à l'IBGN est de 5/20 pour la rivière Gombe et les Oligochètes (Chironomidae) en ont constitué le groupe faunistique indicateur. Cette note est de $11 / 20$ dans la rivière Kinkusa (groupe faunistique indicateur Glossosomatidae). En ce qui concerne la rivière Mangengenge, l'IBGN est de 14/20 (groupe faunistique indicateur: Philopotamidae) (Tableau 7).

Les valeurs de l'indice d'Hilsenhoff ont varié de 7,57 à 8,05 dans la rivière Gombe avec une moyenne de $(7,8 \pm 0,2)$. Elles ont varié de 5,97 à 8,03 dans la rivière Kinkusa avec une moyenne de $(7,6 \pm 1)$. Tandisque dans la rivière Mangengenge, ces valeurs ont oscillé entre 2,13 et 4,8 pour une moyenne de $(3,9 \pm 1,1)$. Les valeurs moyennes obtenues pour les rivières Gombe et Kinkusa sont proches (Tableau 7).

Les valeurs de l'indice d'équitabilité de Pielou calculées pour l'ensemble des 
rivières étudiées ont varié de 0,4 à 0,7 . Elle est de 0,4 dans les rivières Gombe et Kinkusa et elle est de 0,7 dans la rivière Mangengenge.

Les valeurs de l'indice de similarité de Sørensen permettant de comparer les rivières étudiées entre elles, sont reprises dans le Tableau 8. Le coefficient de Sørensen calculé à partir des abondances relatives de la faune benthique de macroinvertébrés de chacune de trois rivières étudiées, indique un haut degré de similarité entre les rivières Gombe et Kinkusa (73\%), une similarité moyenne entre les rivières Kinkusa et Mangengenge $(51 \%)$ et une faible similarité entre les rivières Gombe et Mangengenge (32\%).

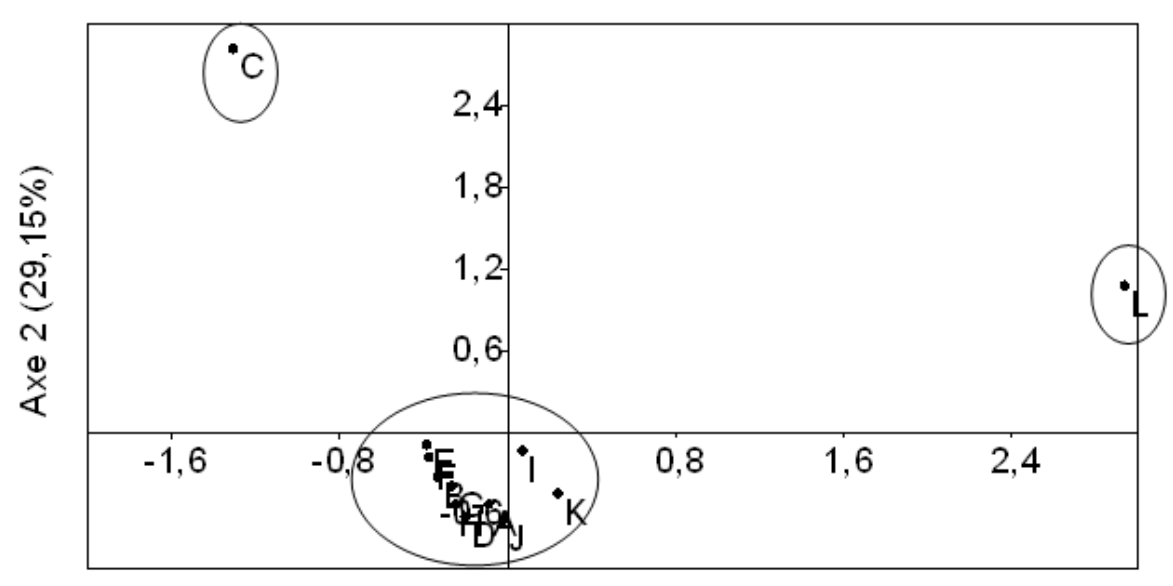

Axe $1(45,36 \%)$

Figure 2 : Ordination des substrats aux lieux de prélèvement des macroinvertébrés benthiques dans les rivières étudiées.

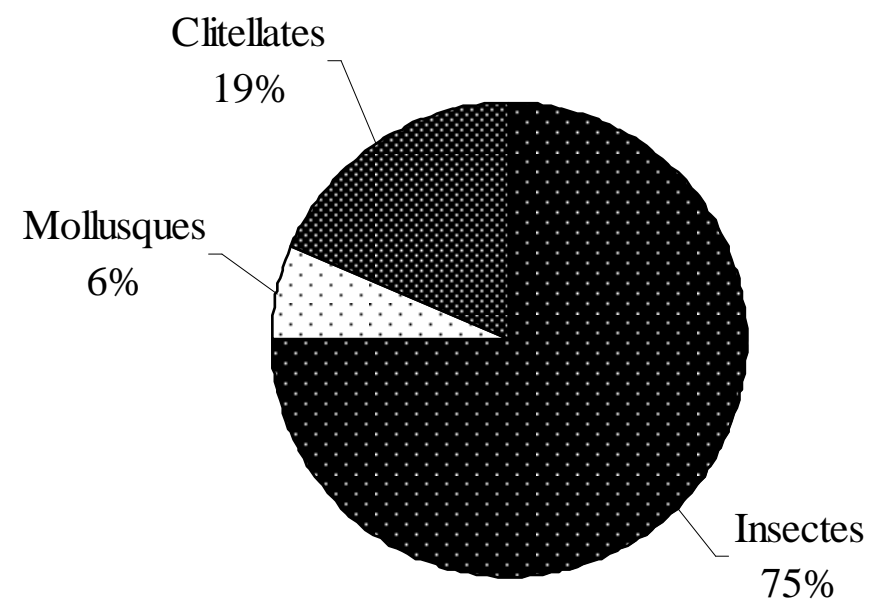

Figure 3 : Proportion relative des macroinvertébrés échantillonnés dans trois hydrosystèmes de Kinshasa. 


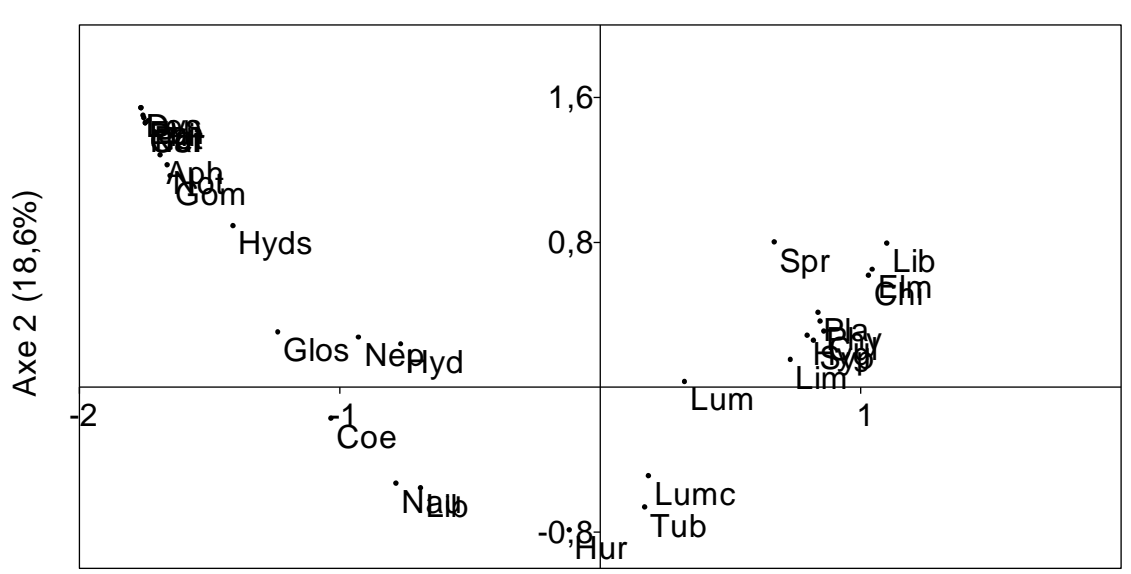

Axe $1(34,6 \%)$

Figure 4 : Correspondance des taxons des macroinvertébrés et des substrats sur le plan des deux premières composantes principales. $\mathrm{V}+\mathrm{MO}(\mathrm{A}) ; \mathrm{V}+\mathrm{S}+\mathrm{MO}$ (B); $\mathrm{V}+\mathrm{MO}+\mathrm{Vg}$ (C); $\mathrm{V}+\mathrm{S}+\mathrm{MO}+\mathrm{G}$ (D) ; $\mathrm{V}+\mathrm{MO}+\mathrm{G}+\mathrm{Ga} \mathrm{E} ; \mathrm{V}+\mathrm{Vg}(\mathrm{F}) ; \mathrm{S}+\mathrm{Vg}(\mathrm{G}) ; \mathrm{S}+\mathrm{MO}(\mathrm{H}) ; \mathrm{S}+\mathrm{MO}+\mathrm{Vg}$ (I); $+\mathrm{V}+\mathrm{Vg}(\mathrm{J}) ; \mathrm{S}+\mathrm{G}+\mathrm{Vg}(\mathrm{K}) ; \mathrm{S}+\mathrm{G}+\mathrm{Vg}+\mathrm{MO}$ (L).

\begin{tabular}{ccccc} 
& & Similarité & & \\
0 & 0 & 0 & 0 & 0 \\
0 & 0 & 0 & 0 \\
0 & 0 & 0 & 0 & 0 \\
0 & 0 & 1 & 0 \\
\hline & 0 & 1 & 1 \\
\hline
\end{tabular}

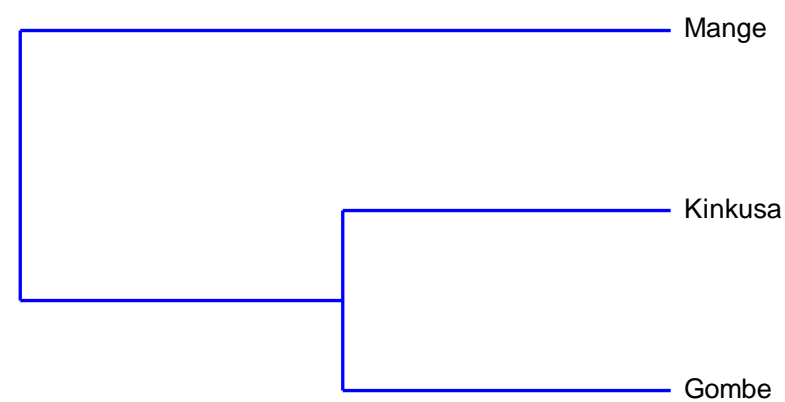

Figure 5 : Dendogramme de similarité de rivières étudiées en fonction de la richesse spécifique en macroinvertébrés benthiques (Mange: Mangengenge).

Tableau 1 : Coordonnées géographiques des stations d'échantillonnages des macroinvertébrés benthiques.

\begin{tabular}{llccc}
\hline Rivières & & Latitude & Longitude & Altitude (m) \\
\hline \multirow{3}{*}{ Gombe } & Crénon & $04^{\circ} 12^{\prime} 53,8^{\prime \prime} \mathrm{S}$ & $15^{\circ} 17^{\prime} 28,9^{\prime \prime} \mathrm{E}$ & 281 \\
\cline { 2 - 5 } Kinkusa & Exutoire & $04^{\circ} 19^{\prime} 11^{\prime \prime} \mathrm{S}$ & $15^{\circ} 16^{\prime} 38,6^{\prime \prime} \mathrm{E}$ & 273 \\
\hline \multirow{2}{*}{ Mangengenge } & Crénon & $04^{\circ} 24^{\prime} 12,2^{\prime \prime} \mathrm{S}$ & $15^{\circ} 14^{\prime} 23,5^{\prime \prime} \mathrm{E}$ & 341 \\
\cline { 2 - 5 } & Exutoire & $04^{\circ} 23^{\prime} 42,8^{\prime \prime} \mathrm{S}$ & $15^{\circ} 13^{\prime \prime} 48,7^{\prime \prime} \mathrm{E}$ & 327 \\
\cline { 2 - 5 } & Crénon & $4^{\circ} 07^{\prime} 39,9^{\prime \prime} \mathrm{S}$ & $15^{\circ} 32^{\prime} 32,2^{\prime \prime} \mathrm{E}$ & 297 \\
\cline { 2 - 5 } & Exutoire & $4^{\circ} 07^{\prime} 45,9^{\prime \prime} \mathrm{S}$ & $15^{\circ} 32^{\prime} 17,6^{\prime \prime} \mathrm{E}$ & 272 \\
\hline
\end{tabular}


Tableau 2: Echelle d'interprétation de l'indice d'Hilsenhoff (Hilsenhoff, 1988).

\begin{tabular}{lcc}
\hline Intervalle des valeurs & Qualité & Etat \\
\hline $0,00-3,75$ & Excellente & Sans pollution organique \\
$3,76-4,25$ & Très bonne & Légère pollution organique possible \\
$4,26-5,00$ & Bonne & pollution organique probable \\
$5,01-5,75$ & Moyenne & pollution organique assez substantielle \\
$5,76-6,50$ & Plutôt mauvaise & pollution organique substantielle \\
$6,51-7,25$ & Mauvaise & pollution organique très substantielle \\
$7,26-10,00$ & Très mauvaise & pollution organique grave \\
\hline
\end{tabular}

Tableau 3: Description des substrats (en \%) dans les stations de prélèvement des macroinvertébrés en saison sèche pour la rivière Gombe.

\begin{tabular}{lccccccccccccc}
\hline Rivières & \multicolumn{1}{c}{ Gombe } & \multicolumn{1}{c}{ Kinkusa } & \multicolumn{1}{c}{ Mangengenge } \\
\hline Types de substrats & I & II & III & IV & I & II & III & IV & V & I & II & III & IV \\
Vase +M.O & 62 & 0 & 0 & 0 & 13 & 33 & 0 & 0 & 0 & 5 & 0 & 0 & 0 \\
Vase +Vg & 0 & 0 & 0 & 75 & 0 & 0 & 0 & 0 & 0 & 0 & 0 & 0 & 0 \\
Vase+MO+Vg & 23 & 80 & 75 & 0 & 0 & 0 & 0 & 0 & 0 & 0 & 0 & 0 & 0 \\
Vase+Sable+MO & 15 & 0 & 0 & 25 & 0 & 0 & 0 & 0 & 0 & 0 & 0 & 0 & 0 \\
Vase+gravier+Galet+MO & 0 & 0 & 25 & 0 & 0 & 0 & 0 & 0 & 0 & 0 & 0 & 0 & 0 \\
Vase+Sable+galet+MO & 0 & 20 & 0 & 0 & 0 & 0 & 0 & 0 & 0 & 0 & 0 & 0 & 0 \\
Sable + vg & 0 & 0 & 0 & 0 & 87 & 33 & 0 & 0 & 0 & 0 & 0 & 0 & 0 \\
Sable +Vase & 0 & 0 & 0 & 0 & 0 & & 66 & 57 & 0 & 0 & 0 & 0 & 0 \\
Sable + MO & 0 & 0 & 0 & 0 & 0 & 11 & 0 & 0 & 54 & 0 & 0 & 0 & 0 \\
Sable +MO+Vg & 0 & 0 & 0 & 0 & 0 & 23 & 34 & 43 & 46 & 0 & 0 & 0 & 0 \\
Grvier+Sable+vg & 0 & 0 & 0 & 0 & 0 & 0 & 0 & 0 & 0 & 95 & 85 & 0 & 0 \\
Gravier+Sable+vg.+MO & 0 & 0 & 0 & 0 & 0 & 0 & 0 & 0 & 0 & 0 & 15 & 100 & 100 \\
\hline
\end{tabular}


Tableau 4 : Liste des macroinvertébrés benthiques des rivières Gombe, Kinkusa et Mangengenge.

\begin{tabular}{|c|c|c|c|c|c|c|c|}
\hline \multirow[b]{2}{*}{ Ordres } & \multirow[b]{2}{*}{ Familles } & \multicolumn{2}{|c|}{ Gombe } & \multicolumn{2}{|c|}{ Kinkusa } & \multicolumn{2}{|c|}{ Mangengenge } \\
\hline & & $\mathbf{N}$ & $n i / \mathbf{N}$ & $\mathbf{N}$ & $\mathrm{ni} / \mathbf{N}$ & $\mathbf{N}$ & ni/N \\
\hline \multirow[t]{5}{*}{ Hémiptères } & Nepidae (Nep) & 2 & 0,00054 & 14 & 0,00781 & 13 & 0,03641 \\
\hline & Aphelocheiridae (Aph) & & 0 & & 0 & 45 & 0,12605 \\
\hline & Gerridae (Ger) & & 0 & & 0 & 2 & 0,0056 \\
\hline & Naucoridae (Nou) & & 0 & 137 & 0,07641 & 36 & 0,10084 \\
\hline & Notonectidae (Not) & & 0 & & 0 & 4 & 0,0112 \\
\hline \multirow[t]{3}{*}{ Odonates } & Coenagrionidae (Coe) & & 0 & 29 & 0,01617 & 22 & 0,06162 \\
\hline & Gomphidae (Gom) & & 0 & & 0 & 24 & 0,06723 \\
\hline & Libellulidae (Lib) & & 0 & 391 & 0,21807 & 108 & 0,30252 \\
\hline \multirow[t]{5}{*}{ Coleopteres } & Hydrophilidae (Hyd) & 11 & 0,00295 & 15 & 0,00837 & 37 & 0,10364 \\
\hline & Sprercheidae (Spr) & 28 & 0,0075 & & 0 & 1 & 0,0028 \\
\hline & Dystiscidae (Dys) & & 0 & & 0 & 1 & 0,0028 \\
\hline & Hygrobiidae (Hyg) & 6 & 0,00161 & 6 & 0,00335 & & 0 \\
\hline & Elmidae (Elm) & 3 & 0,0008 & & 0 & & 0 \\
\hline \multirow[t]{4}{*}{ Diptères } & Ceratopogonidae (Cer) & & 0 & & 0 & 2 & 0,0056 \\
\hline & Culicidae $(\mathrm{Cul})$ & 540 & 0,14473 & 7 & 0,0039 & 1 & 0,0028 \\
\hline & Syrphidae (Syr) & 313 & 0,08389 & 9 & 0,00502 & & 0 \\
\hline & Chironomidae (Chi) & 1352 & 0,36237 & & 0 & & 0 \\
\hline \multirow[t]{7}{*}{ Trichoptères } & Ecnomidae (Ecn) & & 0 & & 0 & 4 & 0,0112 \\
\hline & Hydropsychidae (Hydr) & & 0 & 4 & 0,00223 & 37 & 0,10364 \\
\hline & Leptoceridae (Lep) & & 0 & & 0 & 1 & 0,0028 \\
\hline & Philopotamidae (Phi) & & 0 & & 0 & 3 & 0,0084 \\
\hline & Glossosomatidae (Glo) & & 0 & 3 & 0,00167 & 3 & 0,0084 \\
\hline & Potamanthidae (Pot) & & 0 & & 0 & 3 & 0,0084 \\
\hline & Limnebiidae (Limb) & 59 & 0,01581 & & 0 & 1 & 0,0028 \\
\hline \multirow[t]{6}{*}{ Clitellates } & Lumbricidae (Lumc) & 324 & 0,08684 & 20 & 0,01115 & 4 & 0,0112 \\
\hline & Huridinidae (Hur) & 8 & 0,00214 & 96 & 0,05354 & 2 & 0,0056 \\
\hline & Lumbriculidae (Lum) & 145 & 0,03886 & 89 & 0,04964 & 2 & 0,0056 \\
\hline & Naididae (Nai) & & 0 & & 0 & 1 & 0,0028 \\
\hline & Tubificidae (Tub) & 470 & 0,12597 & 956 & 0,53318 & & 0 \\
\hline & Planariidae (Pla) & 6 & 0,00161 & & 0 & & 0 \\
\hline \multirow[t]{2}{*}{ Gasteropodes } & Physidae (Phy) & 413 & 0,11069 & 16 & 0,00892 & & 0 \\
\hline & Limnaeidae (Lim) & 51 & 0,01367 & 1 & 0,00056 & & 0 \\
\hline Total & 32 & 3731 & 1 & 1793 & 1 & 357 & 1 \\
\hline
\end{tabular}


Tableau 5 : Distribution écologique des macroinvertébrés des rivières étudiées.

\begin{tabular}{|c|c|c|c|c|c|c|c|c|c|c|c|c|c|c|c|c|c|c|c|c|c|c|c|c|c|c|c|c|c|c|c|c|c|c|}
\hline 苋 & 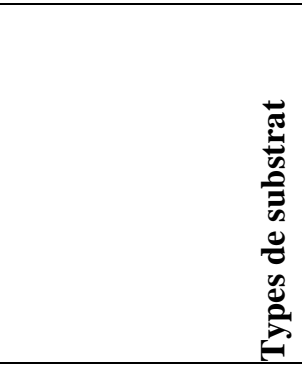 & 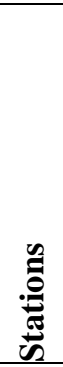 & 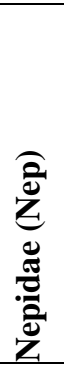 & 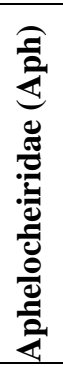 & 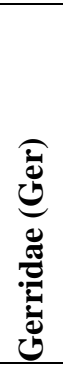 & 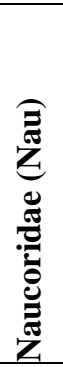 & 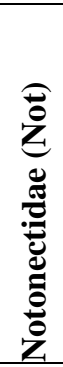 & 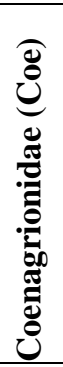 & 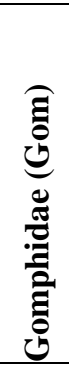 & 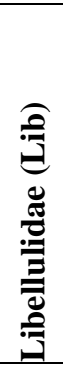 & 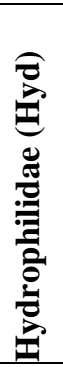 & 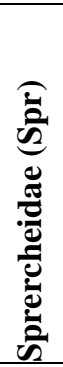 & 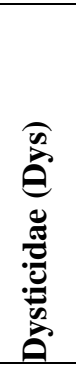 & 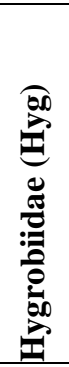 & 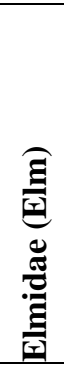 & 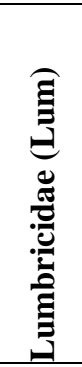 & 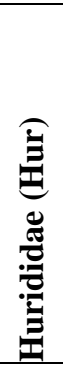 & 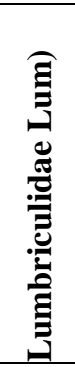 & 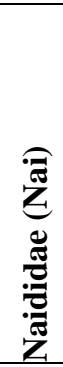 & 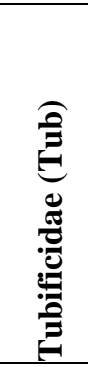 & 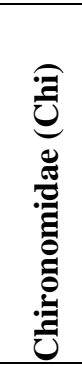 & 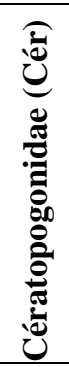 & 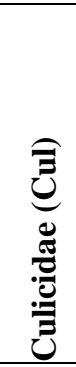 & 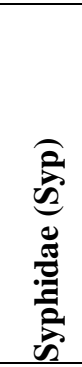 & 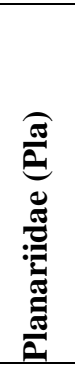 & 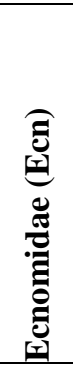 & 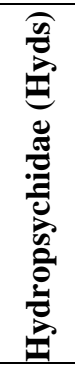 & 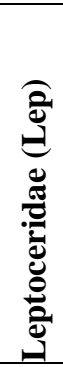 & 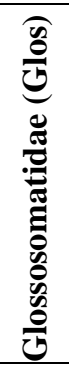 & 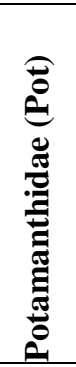 & 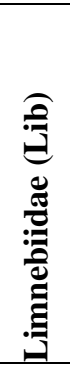 & 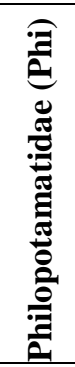 & 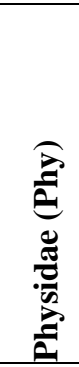 & 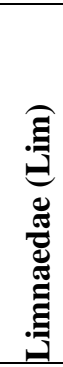 \\
\hline \multirow{9}{*}{ 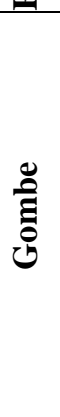 } & $\mathrm{V}+\mathrm{MO}(\mathrm{A})$ & I & & & & & & & & & & & & & & & & & & 4 & 6 & & 2 & 2 & & & & & & & & & 5 & \\
\hline & $\mathrm{V}+\mathrm{S}+\mathrm{MO}(\mathrm{B})$ & I & & & & & & & & & & & & & & 86 & 1 & 15 & & 82 & & & 38 & 2 & & & & & & & & & 1 & 4 \\
\hline & $\mathrm{V}+\mathrm{MO}+\mathrm{Vg}(\mathrm{C})$ & I & & & & & & & & & & & & & 1 & & 1 & 5 & & 89 & 180 & & 105 & 17 & & & & & & & & & 91 & 4 \\
\hline & $\mathrm{V}+\mathrm{MO}+\mathrm{Vg}(\mathrm{C})$ & II & & & & & & & & & 10 & 23 & & & & 227 & 1 & 94 & & 160 & 799 & & 271 & 135 & 6 & & & & & & & & 82 & 42 \\
\hline & $\mathrm{V}+\mathrm{S}+\mathrm{MO}+\mathrm{G}(\mathrm{D})$ & II & & & & & & & & & & & & & & 3 & 1 & 0 & & & 16 & & 31 & & & & & & & & & & 2 & 1 \\
\hline & $\mathrm{V}+\mathrm{MO}+\mathrm{Vg}(\mathrm{C})$ & III & & & & & & & & & & 1 & & 5 & & 1 & 1 & & & 4 & 116 & & 24 & 61 & & & & & & & 28 & & 67 & \\
\hline & $\mathrm{V}+\mathrm{MO}+\mathrm{G}+\mathrm{Ga}(\mathrm{E})$ & III & 2 & & & & & & & & & 4 & & 1 & 1 & 3 & & 1 & & & 119 & & 20 & 54 & & & & & & & 22 & & 43 & \\
\hline & $\mathrm{V}+\mathrm{S}+\mathrm{MO}(\mathrm{B})$ & IV & & & & & & & & & & & & & & 1 & 1 & & & 117 & 5 & & 4 & 18 & & & & & & & 5 & & 8 & \\
\hline & $\mathrm{V}+\mathrm{Vg}(\mathrm{F})$ & IV & & & & & & & & & 1 & & & & 1 & 3 & 2 & 30 & & 14 & 111 & & 45 & 24 & & & & & & & 4 & & 114 & \\
\hline
\end{tabular}


Tableau 5 : Distribution écologique des macroinvertébrés des rivières étudiées (suite).

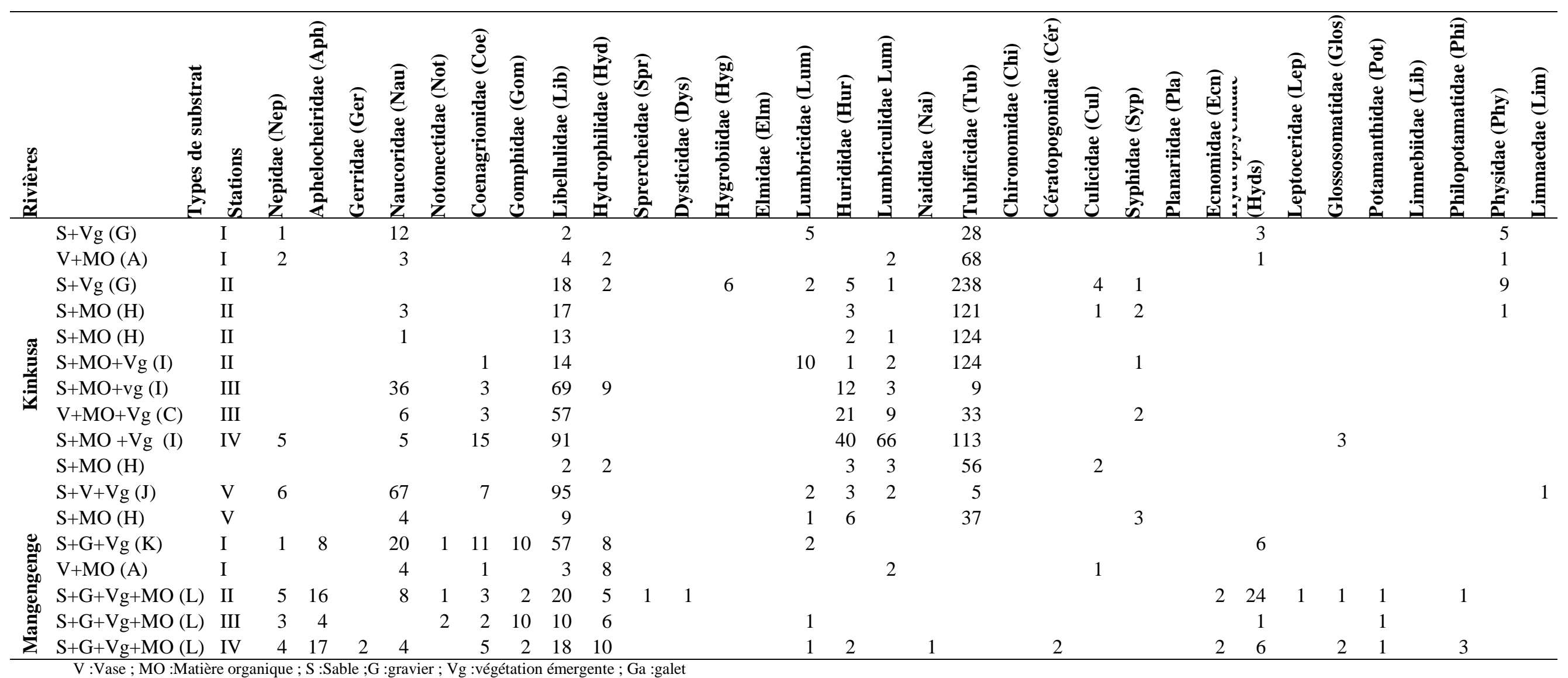


Tableau 6 : Abondance des taxons dans les types de substrats étudiés.

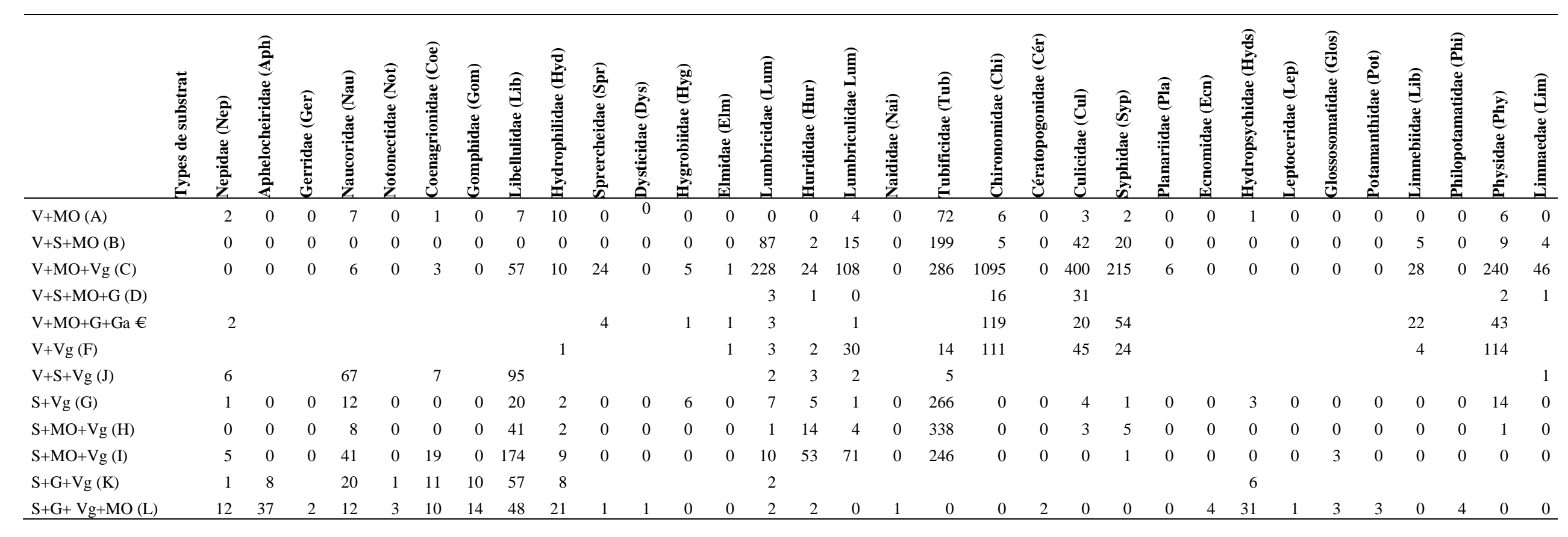

Tableau 7: Valeurs des indices de diversité des rivières étudiées.

\begin{tabular}{lccc}
\hline & Gombe & Kinkusa & Mangengenge \\
\hline H' & 1,4 & 1,8 & 3,3 \\
P & 0,4 & 0,4 & 0,7 \\
IBGN & $5 / 20$ & $11 / 20$ & $14 / 20$ \\
FBI & 7,845 & 7,06 & 3,595 \\
\hline \multicolumn{3}{c}{ H': Indice de Shannon et Weaver ; P : Indice de Piélou ; } \\
\multicolumn{2}{c}{ IBGN : Indice Biologique Global Normalisé ; FBI : Indice d'Hisenhoff). }
\end{tabular}


Tableau 8 : Variation de l'indice de similarité de Sorensen (\%).

\begin{tabular}{|c|c|c|c|c|}
\hline & Gombe & Kinkusa & & Mangengenge \\
\hline Gombe & - & & 3 & 32 \\
\hline Kinkusa & & - & & 51 \\
\hline Mangengenge & & & & - \\
\hline
\end{tabular}

\section{DISCUSSION}

Dans les hydrosystèmes étudiés, nous avons déterminé 12 types de substrats qui sont regroupés en deux catégories: les substrats composés des graviers et ceux composés de vase. Ainsi, les organismes échantillonnés sont soit inféodés à la vase ou alors au gravier. Ce constat rejoint l'assertion faite par Kamb (2013) selon laquelle, la qualité de l'habitat se révèle être une caractéristique déterminante du milieu vis-àvis de la nature, de l'abondance et de la structure des peuplements des macroinvertébrés benthiques d'une station.

La richesse taxonomique observée dans les trois rivières étudiées à Kinshasa, notamment Gombe (16 familles), Kinkusa (16 familles) et Mangengenge (24 familles) est inférieure à celles de la rivière $\mathrm{Nga}$ au Cameroun (Foto et al., 2010). Par contre, la richesse taxonomique cumulée de ces trois cours d'eaux (33 familles) est légèrement supérieure à celle de la rivière Agnéby en Côte d'Ivoire sous climat équatorial, où on dénombre 28 familles (Diomandé, 2009).

Les organismes récoltés dans les rivières Gombe, Kinkusa et Mangengenge en saison sèche sont les insectes (75\%), les Clitellates (19\%) et les gastéropodes (6\%). Parmi les insectes, les Trichoptères sont les mieux représentés dans la rivière Mangengenge (22\% du total des macroinvertébrés benthiques observés). Les études menées par Diomande (2009) et Foto et al. (2010) ont également montré une prédominance des insectes dans la rivière Agnebi et Nga. La composition de la macrofaune benthique des rivières étudiées est proche de celle des eaux douces africaines. Les résultats obtenus corroborent ceux de Diomande et al. (2009) et Foto et al. (2010).

Les Oligochètes échantillonnés dans les trois hydrosystèmes appartiennent à 5 familles dont 4 ont été observées dans la rivière Gombe: (470 Tubificidae, 324 Lumbriculidae, et 145 Lumbricidae). Dans la rivière Kinkusa, les annélides enregistrés appartiennent à 4 familles. Il s'agit des Tubificidae (956 spécimens), Lumbriculidae (89 individus), Lumbricidae (20 spécimens) et Glossiphonidae (3 individus). Ceux capturés dans la rivière Mangengenge appartiennent à 3 familles. Il s'agit des Lumbricidae (4 spécimens), Lumbriculidae ( 2 individus) et Naididae (1 individu). L'abondance des Oligochètes dans la rivière Gombe traduit la mauvaise qualité de l'eau. En effet, les Tubificidae, les Chironomidae et les Syrphidae sont les groupes les plus tolérants à la pollution organique (AFNOR, 2004). Ces organismes sont donc polluorésistants et permettent de conclure que cet hydrosystème est de mauvaise qualité biologique (Camargo et al., 2004; Peterson, 2006).

Les Hémiptères échantillonnés appartiennent à 5 familles. Dans la rivière Gombe, nous avons capturé 2 individus appartenant à la seule famille des Hydropsychidae. Dans la rivière Kinkusa, 118 individus appartenant aux familles de Naucoridae et Nepidae, ont été échantillonnés.

Les Trichoptères récoltés dans l'ensemble des hydrosystèmes appartiennent à 7 familles. Dans la rivière Gombe, aucun Trichoptère n'a été récolté par contre 69 individus ont été récoltés dans la rivière Mangengenge. Une seule famille des Trichoptères (Hydropshychidae) représentée par 4 individus a été observée dans la rivière Kinkusa. Selon Touzin (2008) les Plécoptères, les Trichoptères et les Éphéméroptères sont les groupes les plus sensibles aux polluants. Ces résultats poussent à conclure que la rivière Gombe est plus polluée que les rivières Kinkusa et Mangengenge.

Les substrats observés dans la rivière Gombe contiennent essentiellement de la vase et de la matière organique. Selon Chamley et Deconinck (2013), les concentrations en matière organiques sont plus élevées dans les sédiments fins que dans les sédiments plus grossiers comme le sable. Ce qui justifie une abondance brute plus élevée des macroinvertébrés benthiques dans cette rivière que dans les rivières Kinkusa et Mangengenge; la matière organique étant un des maillons de la chaîne trophique de ces derniers. 
Dans les rivières Gombe et Kinkusa et dans certaines stations étudiées dans la rivière Mangengenge où on observe des substrats vaseux, apparaissent les familles beaucoup plus tolérantes à certaines conditions du milieu comme un déficit en oxygène. Ces taxons appartiennent par exemple aux Chironomidae, Culicidae, Syrphidae (Coimbra et al., 1996). Une telle évolution des taxons est une indication sur la différence des caractéristiques des rivières et des stations qui peut s'expliquer non seulement par la différence d'habitats mais aussi par l'impact des rejets sur le milieu récepteur. Il faut en effet noter que la diversité d'habitats est plus importante dans la rivière Mangengenge que les rivières Gombe et Kinkusa.

Les indices biologiques donnent un aperçu global de l'impact d'une modification du milieu sur les populations d'organismes, même après le passage du flux du polluant (Rodier et al., 2009).

Les valeurs de l'IBGN obtenues pour Gombe : (5/20), Kinkusa: (11/20) et Mangengenge (14/20) permettent de qualifier la Gombe de mauvaise qualité biologique, Kinkusa de qualité passable et Mangengenge de bonne qualité (AFNOR, 2004).

L'indice de Shannon et Weaver calculé pour les trois rivières varie entre 1,6 et 3,4. Il est de 3,4 dans la rivière Mangengenge, 1,7 dans la Gombe et 1,6 dans la rivière Kinkusa. Ces résultats montrent que la rivière Mangengenge a une bonne santé écologique $\left(2 \leq H^{\prime}\right)$ alors que celles des rivières Gombe et Kinkusa sont modérément polluées $(1<H<2$ (Evrard, 1996). Ces résultats s'accordent avec ceux que nous avons obtenus pour l'indice de similarité et regroupent les rivières Gombe et Kinkusa d'un côté et Mangengenge de l'autre.

L’indice d'équitabilité de Piélou calculé pour les rivières Gombe et Kinkusa est respectivement de 0,4 et 0,6 . Tandisque celui de la rivière Mangengenge est de 0,7 . Ces résultats montrent que la répartition des taxons des macroinvertébrés benthiques est plus équilibrée dans la rivière Mangengenge que dans les rivières Kinkusa et Gombe.

Les hydrosystèmes étudiés dans la ville de Kinshasa ainsi que ceux étudiés dans d'autres grandes villes africaines, subissent des pressions anthropiques par le rejet des effluents de diverses natures. Ce constat a également été fait par Gire (2001) et Davis et al. (2011) au Burkina Faso. Ces rejets polluent ces écosystèmes aquatiques et présentent des effets néfastes pour la vie des communautés des macroinvertébrés benthiques (Davis et al., 2011).

\section{Conclusion}

Cette étude a permis de faire un inventaire des peuplements des macroinvertébrés de trois cours d'eaux de la ville de Kinshasa en relation avec leurs habitats respectifs.

Douze types de substrats ont été distingués. Ceux-ci sont des combinaisons de sable, de vase, de végétation émergente, des galets, des graviers et de matière organique. Les substrats composés principalement de vase ont été colonisés par 15 familles de macroinvertébrés et ceux contenant principalement le sable ont été colonisés par 12 familles. Cinq familles ont été recensées dans les deux types principaux de substrats.

Vingt-quatre (24) familles ont été recensés dans la rivière Mangengenge, 16 dans la rivière Kinkusa et 16 dans la rivière Gombe. Ces organismes sont les insectes $(75 \%)$, Clitellates (19\%) et Gastéropodes (6\%).

La richesse taxonomique couplée aux différents indices étudiés, a révélé que la rivière Mangengenge est de bonne qualité écologique, la rivière Kinkusa est d'une qualité écologique moyenne et la rivière Gombe polluée.

Les rivières Gombe et Kinkusa sont proches mais celles-ci sont éloignées de Mangengenge. L'évaluation indiciaire appliquée aux données sur les macroinvertébrés récoltés dans les trois hydrosystèmes montre que les eaux de la rivière Gombe sont de mauvaise qualité biologique, celles de Kinkusa sont de moyenne qualité tandis que celles de Mangengenge sont de bonne qualité. Les valeurs de l'indice de Piélou obtenues montrent que la répartition des taxons des macroinvertébrés est plus équilibrée dans la rivière Mangengenge que dans les rivières Gombe et Kinkusa.

\section{REMERCIEMENTS}

Nous remercions messieurs Rombaud Ndombe, Xavier Kusakana, Edouard Sisa, Eric Lohate, Nasaire Kabemba et Jérémie Amanakou du Département Biologie de l'Université Pédagogique Nationale du Congo pour leur assistance lors des campagnes d'échantillonnage.

\section{REFERENCES}

AFNOR (Association Française de Normalisation). 2004. Essai des eaux. Détermination de l'indice biologique global normalisé (IBGN). Association Française de Normalisation. Norme homologuée. T 90 $350,9 \mathrm{p}$.

Alhou B. 2007. Impact des rejets de la ville de Niamey (Niger) sur la qualité des eaux du fleuve Niger. Thèse de doctorat, Universitaires de Namur, Namur, 230 p. 
Brusle J, Quignard JP. 2004. Les Poissons et leur Environnement Ecophysiologie et Comportements Adaptatifs. Edition TEC et DOC : Londres, Paris, New York ; 1522.

Bultot F. 1954. Saisons et périodes sèches et pluvieuses au Congo belge et au Rwanda-Urundi. Publ. INEAC, Com. $\mathrm{N}^{\circ} 9$ du Bureau climat., coll. In $4^{\circ}$, Bruxelles.

Camargo JA, Alonso A, De la Puente M. 2004. Multimetric assessment of nutrient enrichment in impounded rivers based on benthic macroinvertebrates. Environmental Monitoring and Publishers, 96 : 233-249.

Chamley H, Deconinck JF. 2011. Bases de Sédimentologie ( $3^{\text {ème }}$ édn). Dunod: Paris.

Coimbra CN, Graça MAS, Cortes RM.1996. The effects of a basic effluent on macroinvertebrate community structure in a temporary Mediterranean river. Environmental Pollution, 94: 301-307.

Davis AM, Thorburn PJ, Lewis SE, Bainbridge ZT, Attard SJ, Milla R, Brodie J.E. 2011. Environnemental impacts of irrigated sugarcane production: Herbicide run-off dynamics from farms and associated drainage systems. Agric. Ecosyst. Environ, doi : 10.1016

Diomande D, Kotchi YB, Edia Oi Edia, Konan KF, Gourène G. 2009. Diversité des macroinvertébrés Benthiques de la Rivière Agnéby (Côte d'Ivoire; Afrique de l'Ouest). European Journal of Scientific Research, 33(3): 368-377.

Durand JR, Leveque C. 1981. Flore et Faune Aquatiques de l'Afrique Sahelo-Soudanienne (Tome II). ORSTOM: Paris, France.

Evrard M. 1996. Utilisation des exuviesnymphales de Chironomidae (Diptera) en tant qu'indicateurs biologiques de la qualité des eaux de surface wallonnes. Thèse de Doctorat, Facultés Universitaires Notre Dame de la Paix Namur, Namur, 204 p.

Foto MS, Zebaze TSH, Nyamsi TNL, Ajeagah GA, Njiné T. 2011. Évolution spatiale de la diversité des peuplements de macroinvertébrés benthiques dans un cours d'eau anthropisé en milieu tropical (Cameroun). European Journal of Scientific Research, 55(2): 291-300.

Gire. 2001. État des lieux des ressources en eau du Burkina Faso et de leur cadre gestion. Version finale, Mai 2001. Direction Générale des Ressources en Eau. Ouagadougou, Burkina Faso.

Hammer Ø, Harper DAT, Ryan PD 2001. PAST: Paleontological Statistics Software Package for Education and Data Analysis. Palaeontologia Electronica 4(1): 9p.
Hilsenhoff WL. 1988. Rapid field assessment of organic pollution with a family-level biotic index. J. N. Am. Benthol. Soc., 7(1): 65-68.

Kamb TJ-C. 2013. Etude de la structure et de la dynamique des peuplements des Macroinvertébrés benthiques d'un système lotique : Cas de la rivière Lukunga Kinshasa / R.D. Congo Mémoire de DEA, Université Pédagogique Nationale (UPN), Kinshasa, 80 p.

Kuyitila LP. 2011. Diversité des macroinvertébrés des rivières N'sele et Mango en rapport avec la qualité de leurs eaux. Mémoire, Université de Kinshasa, Kinshasa, 27p.

Legendre P, Legendre L. 1998. Écologie numérique. 2e édn. Elsevier Science BV : Amsterdam, Pays-bas.

Lelo NF. 2008. Kinshasa, Ville et Environnement. Edition. L'Harmattan : Kinshasa ; 282 p.

Mangaya NP. 2010. Utilisation des macroinvertébrés benthiques dans l'évaluation de la qualité de l'eau de surface : Cas de la rivière Tshwenge à Kinshasa. Mémoire, Université de Kinshasa, Kinshasa, $52 \mathrm{p}$.

Mbadu ZV. 2002. Le bassin versant de la rivière Lukunga, impact de son utilisation sur son environnement, mémoire de DESS, ERAIFT, Kinshasa, 138 p.

MDDEFP (Ministère du Développement Durable, de l'Environnement, de la Faune et des Parcs) 2013. Guide de surveillance biologique basée sur les macroinvertébrés benthiques d'eau douce du Québec - Cours d'eau peu profonds à substrat grossier, 2013. Direction du suivi de l'état de l'environnement, ISBN 978-2-550-69169-3 (PDF), 2e édition, $88 \mathrm{p}$.

Moisan J, Pelletier L. 2008. Guide de surveillance biologique basée sur les macroinvertébrés benthiques d'eau douce du Québec - Cours d'eau peu profonds à substrat grossier, 2008. Direction du suivi de l'état de l'environnement, ministère du Développement durable, de l'Environnement et des Parcs, Québec, Canada.

Moisan J. 2010. Guide d'identification des principaux macroinvertébrés benthiques d'eau douce du Québec, 2010 - Surveillance volontaire des cours d'eau peu profonds, Direction du suivi de l'état de l'environnement, Ministère du 
Développement Durable, de l'Environnement et des Parcs, Québec, Canada,82 p.

Muli JR, Mavuti KM. 2001. The benthic macrofauna community of Kenyan waters of Lake Victoria. Hydrobiologia, 458 : 83-90.

Peterson M. 2006. Course materials : Biology 326, ecology lab. Western Washington University, Department of biology, Bellingham, Washington.

Piscart C, Moreteau JC, Beise JN. 2005. Biodiversity and structure of macroinvertebrate communities along a small permanent salinity gradient (Meurthe River, France). Hydrobiologia, 551: 227-236.

Puta KE. 2005. Contribution à l'étude de la qualité des eaux des rivières de Kinshasa: cas de la rivière Kwambila. Mémoire, Université de Kinshasa, Kinshasa, 32p.

Rodier J, Legure B, Merlet N. 2009. L'Analyse de l'Eau ( $9^{\text {ème }}$ édn). Dunod: Paris.

Sørensen TA. 1948. The method of establishing groups of equal amplitude in plant sociology based on similarity of species content, and its application to analyses of the vegetation on Danish commons. Kongelige Danske Videnskabernes Selskabs Biologiske Skrifter, 5: 1-34.

Tachet H, Richoux P, Bournaud M, Usseglio-Pola tera P. 2010. Invertébrés d'Eau Douce. Systématique, Biologie, Ecologie, CNRS: Paris.

Touzin D. 2008. Utilisation des macroinvetébrés benthiques pour évaluer la dégradation de la qualité de l'eau des rivières au Québec, Mémoire inédit. Université de Québec, Canada.

Tubi NE. 2005. Contribution à l'étude de la qualité des eaux des rivières de Kinshasa: cas de la rivière BUMBU. Mémoire, Université de Kinshasa, Kinshasa, 44p. 